University of Nebraska - Lincoln

DigitalCommons@University of Nebraska - Lincoln

$7-24-2000$

\title{
Static-Electric-Field-Induced Polarization Effects in Harmonic Generation
}

Bogdan Borca

University of Nebraska - Lincoln

A.V. Flegel

Voronezh State University, Voronezh, Russia

M.V. Frolov

Voronezh State University, Voronezh, Russia

N. L. Manakov

Voronezh State University, manakov@phys.vsu.ru

Dejan B. Milosevic

University of Nebraska - Lincoln

See next page for additional authors

Follow this and additional works at: https://digitalcommons.unl.edu/physicsstarace

Part of the Physics Commons

Borca, Bogdan; Flegel, A.V.; Frolov, M.V.; Manakov, N. L.; Milosevic, Dejan B.; and Starace, Anthony F., "Static-Electric-Field-Induced Polarization Effects in Harmonic Generation" (2000). Anthony F. Starace Publications. 75.

https://digitalcommons.unl.edu/physicsstarace/75

This Article is brought to you for free and open access by the Research Papers in Physics and Astronomy at DigitalCommons@University of Nebraska - Lincoln. It has been accepted for inclusion in Anthony F. Starace Publications by an authorized administrator of DigitalCommons@University of Nebraska - Lincoln. 


\section{Authors}

Bogdan Borca, A.V. Flegel, M.V. Frolov, N. L. Manakov, Dejan B. Milosevic, and Anthony F. Starace 


\title{
Static-Electric-Field-Induced Polarization Effects in Harmonic Generation
}

\author{
Bogdan Borca, ${ }^{1, *}$ A. V. Flegel, ${ }^{2}$ M. V. Frolov, ${ }^{2}$ N. L. Manakov, ${ }^{2}$ Dejan B. Milošević, ${ }^{1, \dagger}$ and Anthony F. Starace ${ }^{1}$ \\ ${ }^{1}$ Department of Physics and Astronomy, The University of Nebraska, Lincoln, Nebraska 68588-0111 \\ ${ }^{2}$ Department of Physics, Voronezh State University, 394693 Voronezh, Russia
}

(Received 22 December 1999)

\begin{abstract}
Two static-electric-field-induced effects on harmonic generation are demonstrated analytically and numerically: elliptic dichroism (in which the harmonic yield is different for right and left elliptically polarized laser fields) and elliptical polarization of harmonics produced by linearly polarized driving laser fields. Both effects stem from interference of real and imaginary parts of the nonlinear atomic susceptibilities. Possibilities for experimentally measuring these effects are discussed.
\end{abstract}

PACS numbers: $42.65 . \mathrm{Ky}, 32.80 . \mathrm{Qk}, 32.80 . \mathrm{Wr}, 42.50 . \mathrm{Hz}$

High-order harmonic generation (HHG) has become a main topic of intense laser-atom physics [1]. Both to understand the HHG process better and to enhance its use in applications, many studies have focused on the control of HHG [1]. Among the most recent are those concerned with the polarization characteristics of the harmonics. Weihe et al. [2] showed that the polarization ellipse of the emitted harmonics may rotate with respect to that of the incident laser polarization ellipse. Reference [3] showed that this unusual effect stems from interference between the real and imaginary parts of the corresponding nonlinear susceptibilities. Burnett et al. [4] found that the polarization characteristics of emitted harmonics vary greatly along the harmonic plateau. Antoine et al. [5] confirmed such behavior, finding that the rotation angles and ellipticities of the harmonics strongly depend on their position in the spectrum, but that the ellipticities of the harmonics remain smaller than that of the driving laser. Other experiments have found unusual polarization characteristics for particular harmonics [6]. Theorists, meanwhile, have found that prediction of harmonic polarization provides a stringent test of models and methods $[3,7,8]$.

Other studies have focused on the use of a strong static electric field to control the intensities and plateau structure of HHG [9-11]. A static electric field allows the generation of even harmonics, control of the intensity of particular harmonics [9], and the appearance of high-order plateau structures [10]. Wang et al. [12] examined the effect on HHG intensities of elliptically polarized laser light and a static electric field parallel to the laser polarization ellipse's major axis. They found that circularly polarized light generates harmonics (which is consistent with the three-step model [13], since the static field reflects electronic wave packets back to the atomic core). They did not, however, examine the polarization of the harmonics.

We present here some results of a general formulation of the problem of HHG for arbitrarily polarized laser light in the presence of an arbitrarily oriented static electric field. We show that a static electric field has striking effects on the polarization of high-order harmonics and on the dependence of harmonic yield on the laser field ellipticity. We demonstrate that (i) in the presence of a static electric field the harmonics are in general elliptically polarized, even for a linearly polarized driving laser (which contrasts with results obtained in the absence of a static field [5]), and (ii) the static electric field leads to a significant elliptic dichroism effect; i.e., the intensity of a harmonic differs substantially for right and left helicities of an elliptically polarized laser beam. These results demonstrate the significant control a static electric field permits of polarization effects in HHG.

We consider a single atom interacting with a laser field of arbitrary polarization, i.e.,

$$
\mathbf{F}(\mathbf{r}, t)=F \operatorname{Re}\{\mathbf{e} \exp [i(\mathbf{k} \cdot \mathbf{r}-\omega t)]\},
$$

where the complex polarization vector $\mathbf{e}$ is defined in a coordinate-frame-invariant way using the laser field wave vector $\mathbf{k}$ and the unit vector $\hat{\boldsymbol{\varepsilon}}$ along the major axis of the laser polarization ellipse,

$$
\mathbf{e}=\frac{\hat{\boldsymbol{\varepsilon}}+i \eta(\hat{\mathbf{k}} \times \hat{\boldsymbol{\varepsilon}})}{\sqrt{1+\eta^{2}}}, \quad-1 \leq \eta \leq+1 .
$$

Here $\eta$ is the ellipticity, where $\eta=+1(-1)$ corresponds to right (left) circular polarization, and $\eta=0$ to linear polarization. In terms of these vectors, the degree of linear polarization is given by $l=\mathbf{e} \cdot \mathbf{e}=\left(1-\eta^{2}\right) /\left(1+\eta^{2}\right)$, and the degree of circular polarization by $\xi=i \hat{\mathbf{k}} \cdot(\mathbf{e} \times$ $\left.\mathbf{e}^{*}\right)=2 \eta /\left(1+\eta^{2}\right)$, both of which are simply related to the usual Stokes parameters $S_{1}, S_{2}, S_{3}$ (i.e., $\xi=S_{2}$, $l=\sqrt{S_{1}^{2}+S_{3}^{2}}$ ) [14]. The static electric field $\mathcal{F}$ is oriented along the direction $\hat{\boldsymbol{e}}_{0}$, which we assume in the following to lie in the plane of the laser polarization ellipse, perpendicular to $\mathbf{k}$.

Complete information concerning the intensity and polarization properties of the $n$th harmonic may be extracted from the amplitude $A_{n \omega}\left(\mathbf{e}^{\prime}\right)$ describing dipole emission of a harmonic (with frequency $\omega^{\prime}=n \omega$, measured polarization $\mathbf{e}^{\prime}$, and propagation direction $\hat{\mathbf{k}}^{\prime}=\hat{\mathbf{k}}$ ) by an atom in the presence of fields $\mathcal{F}$ and $\mathbf{F}$, where

$$
A_{n \omega}\left(\mathbf{e}^{\prime}\right)=\mathbf{e}^{\prime *} \cdot \tilde{\mathbf{d}}_{n},
$$

and $\tilde{\mathbf{d}}_{n}$, defined in Eq. (9), is a (complex) matrix element of the dipole operator. Taking into account, as in [3], the spatial and temporal symmetry properties of the vectors 
involved [i.e., $\mathbf{F}(\mathbf{r}, t), \mathcal{F}, \mathbf{e}, \mathbf{e}^{*}$, and $\tilde{\mathbf{d}}_{n}$ ], as well as the fact that only the part of $\tilde{\mathbf{d}}_{n}$ transverse to $\hat{\mathbf{k}}$ contributes to Eq. (3), one may express $\tilde{\mathbf{d}}_{n}$ in general as

$$
\tilde{\mathbf{d}}_{n}=\chi_{1} \mathbf{e}+\chi_{2} \mathbf{e}^{*}+\chi_{3} \hat{\boldsymbol{e}}_{0} \text {. }
$$

$$
\left|\tilde{\mathbf{d}}_{n}\right|^{2}=\sum_{i=1}^{3}\left|\chi_{i}\right|^{2}+\sqrt{2(1+l)} \operatorname{Re}\left[\left(\chi_{1}+\chi_{2}\right) \chi_{3}^{*}\right] \cos \varphi
$$

The last term in Eq. (5), involving $\xi$, exhibits elliptic dichroism: it has opposite signs for right $(\xi>0)$ and left $(\xi<0)$ elliptic polarization of the laser field. Obviously this term vanishes for $\xi=0$ (linear laser polarization). It vanishes also for $|\xi|=1$ (circularly polarized laser light). This may be understood on symmetry grounds: the pseudoscalar $\xi$ can only enter $\left|\tilde{\mathbf{d}}_{n}\right|^{2}$ multiplied by another pseudoscalar quantity, namely, $\sin \varphi=\hat{\boldsymbol{e}}_{0} \cdot(\hat{\mathbf{k}} \times \hat{\boldsymbol{\varepsilon}})$. For circular polarization, the direction of $\hat{\boldsymbol{\varepsilon}}$ is arbitrary and no other preferential direction in the polarization plane exists besides that of the static field $\left(\hat{\boldsymbol{e}}_{0}\right)$; since no additional pseudoscalar exists, the dichroic term must vanish. A detailed analysis of the $l$ and $\varphi$ dependence of the susceptibilities $\chi_{i}$, e.g., in terms of higher order perturbative expansions in $F$ (cf. [3]), shows that for $l \rightarrow 0$, the last term of Eq. (5) is proportional to $l \xi \sin (2 \varphi)$ and hence vanishes. Similar considerations imply that when $\varphi=$ $\pi / 2$ the elliptic dichroism term also vanishes. Therefore, when $0<|\xi|<1$ and $0<\varphi<\pi / 2$, elliptic dichroism may be observed, caused by an interference between the real and imaginary parts of $\chi_{1,2}$ and the static-electricfield-induced susceptibility, $\chi_{3}$.

The polarization properties of the $n$th harmonic are described by its Stokes parameters $S_{i}^{n}, 1 \leq i \leq 3$ [14]. These are defined in terms of the intensity of the $n$th harmonic having a detected polarization $\mathbf{e}^{\prime}: I_{n \omega}\left(\xi^{\prime}, \theta\right) \propto$ $\left|A_{n \omega}\left(\mathbf{e}^{\prime}\right)\right|^{2}$, where $\theta$ is the angle between the directions $\hat{\boldsymbol{\varepsilon}}$ and $\hat{\boldsymbol{\varepsilon}}^{\prime}$ of the major axes of the polarization ellipses, respectively, of the laser and of the detected harmonic. Thus $S_{1}^{n}$ equals the difference between $I_{n \omega}\left(\xi^{\prime}=0, \theta\right)$ for $\theta= \pm \pi / 4$ divided by the sum. Similarly $S_{2}^{n}$ involves $I_{n \omega}\left(\xi^{\prime}= \pm 1, \theta\right)$, and $S_{3}^{n}$ involves $I_{n \omega}\left(\xi^{\prime}=0, \theta\right)$ for $\theta=0, \pi / 2$. For a linearly polarized laser, Eq. (4) shows that there are only two independent susceptibilities, $\chi_{\|}=$ $\chi_{1}+\chi_{2}+\chi_{3} \cos \varphi$ and $\chi_{\perp}=\chi_{3} \sin \varphi$. One easily finds that the harmonic's intrinsic degree of circular polarization, $\xi_{n}$, and offset angle, $\theta_{n}$, are

$$
\begin{gathered}
\xi_{n} \equiv S_{2}^{n}=\frac{2 \operatorname{Im}\left(\chi_{\perp} \chi_{\|}^{*}\right)}{\left|\chi_{\|}\right|^{2}+\left|\chi_{\perp}\right|^{2}}, \\
\tan 2 \theta_{n} \equiv \frac{S_{1}^{n}}{S_{3}^{n}}=\frac{2 \operatorname{Re}\left(\chi_{\perp} \chi_{\|}^{*}\right)}{\left|\chi_{\|}\right|^{2}-\left|\chi_{\perp}\right|^{2}} .
\end{gathered}
$$

As for elliptic dichroism, the ellipticity $\xi_{n}$ originates from an interference of the real and imaginary parts of the nonlinear susceptibilities and may be observed for $0<\varphi<$ $\pi / 2$. In contrast, the offset angle originates from the obvious anisotropy of the atom in a static field and is nonzero even for real $\chi_{\|}$and $\chi_{\perp}$.
The complex scalars $\chi_{1,2,3}$ are the nonlinear susceptibilities, which depend only on nonvector parameters (e.g., $\omega$, $F, \mathcal{F}, l)$ and on the angle $\varphi$ between $\hat{\boldsymbol{\varepsilon}}$ and $\hat{\boldsymbol{e}}_{0}$. The total intensity of the $n$th harmonic, summed over its polarization states, is proportional to $\left|\tilde{\mathbf{d}}_{n}\right|^{2}$, where

$+2 l \operatorname{Re}\left(\chi_{1} \chi_{2}^{*}\right)-\xi \sqrt{2 /(1+l)} \operatorname{Im}\left[\left(\chi_{1}-\chi_{2}\right) \chi_{3}^{*}\right] \sin \varphi$

The above symmetry analysis is independent of any dynamical model, but quantitative estimations of the magnitude of the effects require numerical calculations of the amplitudes in Eq. (3). An accurate, ab initio way to calculate them nonperturbatively is to use the quasistationary, quasienergy states (QQES) or non-Hermitian Floquet states (see, e.g., [15]) for an atom in strong laser and static electric fields, $\Phi_{\varepsilon}(\mathbf{r}, t)$, where $\varepsilon$ is the complex quasienergy. Because of their asymptotically divergent terms in $r$ (in the open ionization channels), the QQES wave functions are not normalizable in the standard way; thus matrix elements of the dipole emission operator $\mathbf{r}$ between QQES functions diverge when calculated in the usual way. Instead, properly normalized (dual) functions, $\tilde{\Phi}_{\varepsilon}(\mathbf{r}, t)$, must be used as bra vectors in a calculation of transition matrix elements [16], as when using quasistationary states in radiationless problems (see, e.g., [17]). In the presence of two fields, $\mathcal{F}$ and $\mathbf{F}(\mathbf{r}, t)$ with an arbitrary elliptical polarization, the proper dual functions are given by

$$
\tilde{\Phi}_{\varepsilon}(\mathbf{r}, t)=\left[\Phi_{\varepsilon}(\mathbf{r},-t ; \eta \rightarrow-\eta)\right]^{*} .
$$

For $\eta=0$, the dual function in Eq. (8) coincides with that introduced by Potvliege and Shakeshaft [16] for a linearly polarized laser field. The time-dependent (complex) dual dipole moment is thus calculated as

$$
\tilde{\mathbf{d}}(t)=\left\langle\tilde{\Phi}_{\varepsilon}(\mathbf{r}, t)|\mathbf{r}| \Phi_{\varepsilon}(\mathbf{r}, t)\right\rangle=\frac{1}{2} \sum_{n} \tilde{\mathbf{d}}_{n} e^{-i n \omega t},
$$

using $\tilde{\Phi}_{\varepsilon}(\mathbf{r}, t)$ as the bra vector. The $n$th Fourier coefficient, $\tilde{\mathbf{d}}_{n}$, of Eq. (9) is then used in Eq. (3) for the generation amplitude.

Based on this QQES approach, we calculated the susceptibilities $\chi_{1,2,3}$ in Eq. (4) using a three-dimensional zero-range potential model for the atom. The QQES solution for this model [18] has been used in recent HHG calculations (see, e.g., $[9,10,19])$. We note that if (as done here) the quasienergy $\varepsilon$ is approximated by the unperturbed binding energy, $E_{0}$, of the model atom and if all but the leading Fourier coefficients of $\Phi_{E_{0}}(\mathbf{r}, t)$ at the origin $(r \rightarrow 0)$ are neglected, as in [19], then our approximate QQES result for the amplitude $A_{n \omega}\left(\mathbf{e}^{\prime}\right)$ coincides with the result of the $S$-matrix approach $[7,10,20]$ provided that the latter takes into account the so-called continuumcontinuum term [7]. For HHG by an elliptically polarized laser in the presence of a static electric field, each susceptibility $\chi_{1,2,3}$ involves an infinite sum of one-dimensional time integrals of a product of Bessel functions. These integrals were evaluated numerically. In order to make our 
numerical results applicable to a variety of atoms and field parameters, we adopt scaled units: our energy unit is the atom binding energy $\left|E_{0}\right|$, and our electric field strength unit is the internal field, $F_{0}=\sqrt{2 m\left|E_{0}\right|^{3}} / e \hbar$. The displayed results are calculated for $\hbar \omega=0.2, F=0.2$, and $\mathcal{F}=10^{-3}$, all in scaled units, as a particular numerical example.

Figure 1 shows the HHG spectrum for different values of the laser field ellipticity, $\eta$, when the static field is collinear with the polarization direction, $\hat{\boldsymbol{\varepsilon}}$. As $\eta$ increases, the intensity of the harmonics decreases, but they are still produced even for a circularly polarized laser field (as shown by [12]). As $\eta$ increases, the gap between even and odd harmonic intensities decreases. For right-circular polarization (i.e., $\eta=1.0$ ) both odd and even harmonics are produced only due to the static field; thus they have the same levels of intensity.

In Fig. 2 we predict elliptic dichroism for both even and odd harmonics as a function of the angle $\varphi$ between the directions of the major axis of the laser polarization ellipse, $\hat{\boldsymbol{\varepsilon}}$, and the static electric field, $\hat{\boldsymbol{e}}_{0}$. If we denote by $I_{+}$and $I_{-}$the intensities obtained for laser ellipticities $\pm|\eta|$, then the dichroic ratio $\delta=\left(I_{+}-I_{-}\right) /\left(I_{+}+I_{-}\right)$ is a good measure of this effect. Our calculations show that significant values of $\delta$ appear as a result of either outof-phase oscillations of $I_{+}$and $I_{-}$, or in-phase oscillations with, e.g., $I_{+}>I_{-}$. One sees from Fig. 2 that $\delta$ is significant for both even and odd harmonics, that it is significant throughout the plateau region, and that it is very sensitive to both $|\eta|$ and $\varphi$.

Figure 3 shows the circular polarization degree, $\xi_{n}$, for low-order even harmonics produced by a linearly polarized laser as a function of the angle $\varphi$ [cf. Eq. (6)]. For $\mathcal{F}=0$, a linearly polarized laser field generates only linearly polarized odd harmonics. As our static field strength is very small compared to the laser field strength, it does not change the polarization of the odd harmonics significantly (e.g., we found $\left|\xi_{n}\right|<0.03$ for $n=3,5,7$ ). How-

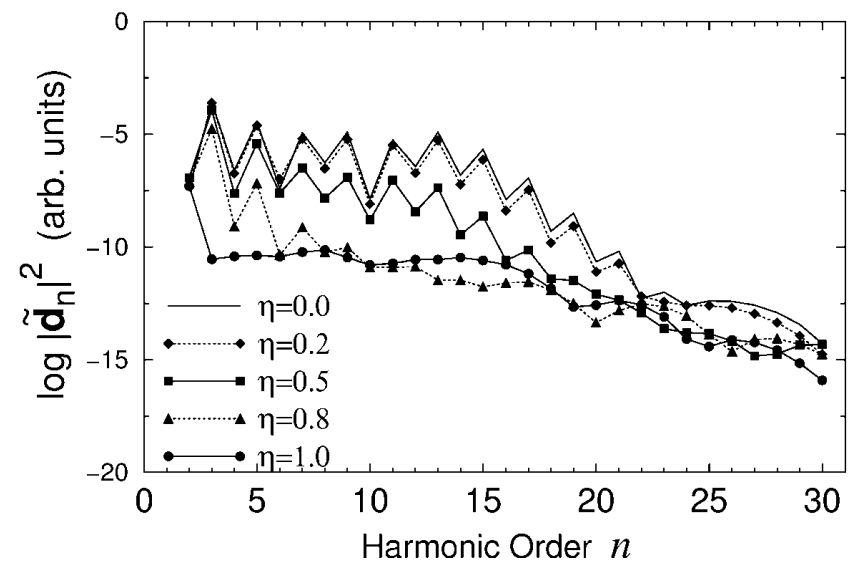

FIG. 1. Harmonic intensities in the presence of a static field parallel to the major axis of the laser polarization ellipse, for five laser ellipticity values $\eta$. In scaled units (see text), $\omega=0.2$, $F=0.2$, and $\mathcal{F}=10^{-3}$. ever, the even order harmonics, which owe their presence to the static field, are strongly affected by its orientation. It is even possible to produce harmonics with polarizations that are very close to circular over a fairly large range of $\varphi$ [cf. Fig. 3 for $n=2$ and 6 and $60^{\circ} \leq \varphi \leq 70^{\circ}$ ]. As the harmonic order increases, the domain of significant nonlinear polarization becomes narrower and is increasingly shifted towards $\varphi=90^{\circ}$ (as one can see for the 8th harmonic). When $\varphi=90^{\circ}$, all harmonics are emitted with linear polarization but with different orientations: odd harmonics along $\hat{\boldsymbol{\varepsilon}}$ and even ones along $\hat{\boldsymbol{e}}_{0}$.

Our analysis above [cf. (5) and (6)] shows that the polarization effects demonstrated in Figs. 2 and 3 stem from the anisotropy induced by the static field [cf. Eq. (4)] in an absorptive medium. We may extend this analysis by noting that the anisotropy can be induced by means other than a static electric field. For example, a similar effect may appear in harmonics generated by reflection of an intense laser beam by a metal surface, where the anisotropy is introduced by the surface normal vector. As another example, closer to the one we have treated, the required anisotropy may be introduced by a second, low-intensity, linearly polarized laser beam collinear with the first, $\mathbf{F}_{\Omega}(t)=F_{\Omega} \hat{\boldsymbol{e}}_{0} \cos \Omega t$. In this case, if one considers only the harmonics of the high-intensity laser, Eqs. (3)-(7) have exactly the same form and therefore lead to the same polarization effects. For a low frequency, $\Omega$, neglecting (in lowest approximation) the (weak) $\Omega$ dependence of the susceptibilities $\chi_{i}$, the only effect is to replace the static field amplitude $\mathcal{F}$ by an "effective amplitude" of order $F_{\Omega}$ [21]. Though we have presented our analysis and numerical results for the simplest conceptual case, that of HHG in the presence of a static field, we emphasize by these additional examples a major goal of our paper: to demonstrate how the introduction of a second polar vector in the problem [in addition to $\mathbf{F}(t)$ ] leads to interference between the complex susceptibilities $\chi_{i}$, the results of which are unusual polarization properties

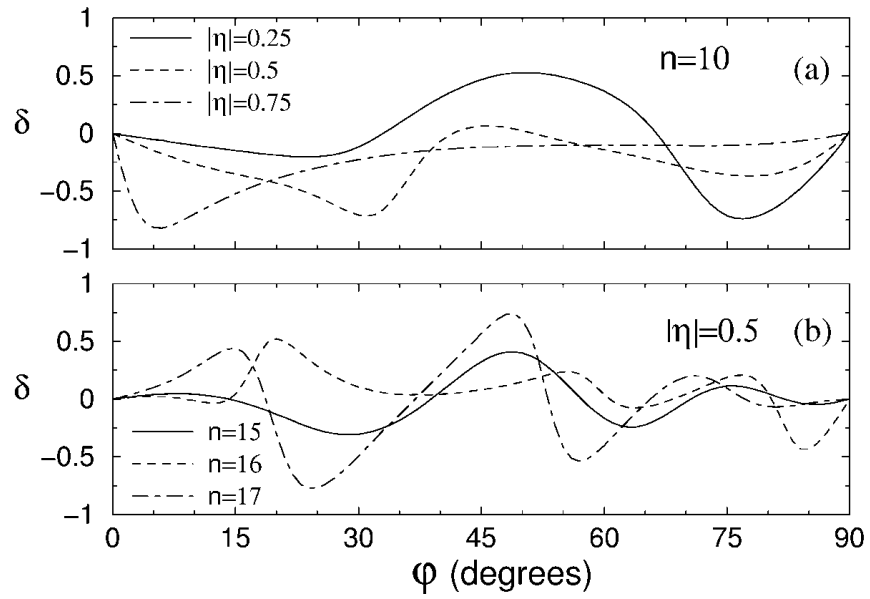

FIG. 2. Elliptic dichroism parameter $\delta=\left(I_{+}-I_{-}\right) /\left(I_{+}+\right.$ $I_{-}$) for (a) $n=10$ and $|\eta|=0.25,0.5$, and 0.75 , and (b) $n=$ 15,16 , and 17 , and $|\eta|=0.5$, and $\omega, F$, and $\mathcal{F}$ as in Fig. 1 . 


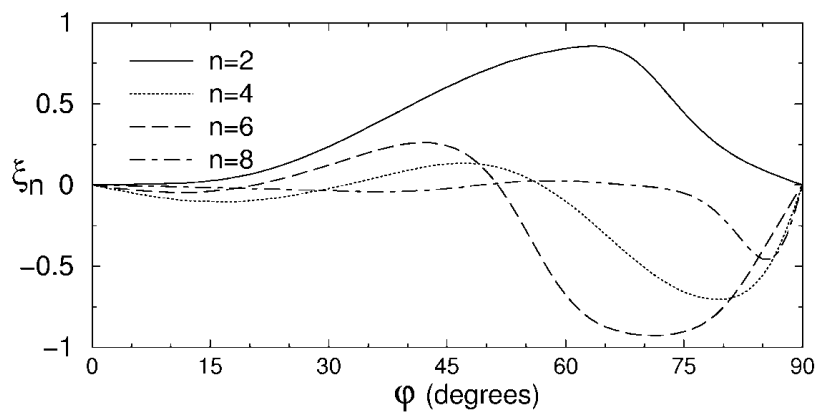

FIG. 3. Circular polarization degree, $\xi_{n}$, for even harmonics ( $n=2,4,6$, and 8$)$ produced by a linearly polarized laser field for different angles $\varphi$. Parameter values $\omega, F$, and $\mathcal{F}$ are as in Fig. 1.

of the generated harmonics from an initially isotropic and absorptive medium. The predicted effects depend only on the magnitudes of the real and imaginary parts of the susceptibilities $\chi_{i}$. Physically, the imaginary parts are connected with the ionization of the target atom by the laser field, and hence they are zero if we neglect this ionization. The polarization effects can therefore be called ionization(or dissipation-) induced effects.

Experimental verification of these harmonic polarization effects depends on the qualitative matching of our scaled parameters with a particular atomic system and a particular set of field strengths and frequencies. For example, for a Xe atom, the scaled parameters employed in Figs. 1-3 correspond to a laser with $\lambda=511.5 \mathrm{~nm}$ and intensity $2.48 \times 10^{14} \mathrm{~W} / \mathrm{cm}^{2}$, which are close to typical values for a frequency-doubled Nd:YAG laser $(\lambda=532 \mathrm{~nm})$. The static field strength, however, is $2.16 \mathrm{MV} / \mathrm{cm}$, which is far higher than typical laboratory static fields even though it is weak compared with the laser field $(\mathcal{F} / F=5 \times$ $\left.10^{-3}\right)$. The requisite field strength ratio may nevertheless be achieved in a two color experiment in which the weak laser field, $\mathbf{F}_{\Omega}(t)$, has a much lower frequency, $\Omega$, than that of the intense laser. For example, the frequency of a $\mathrm{CO}_{2}$ laser (scaled frequency $\Omega=0.009$ for $\mathrm{Xe}$ ) is 22 times smaller than that of a frequency-doubled Nd:YAG laser, while the necessary $\mathcal{F} / F$ ratio could be achieved with an intensity of order $6 \times 10^{9} \mathrm{~W} / \mathrm{cm}^{2}$. The weak laser field may be regarded as approximately constant compared with the strong laser field and hence measured polarization results in such an experiment would be qualitatively similar to those predicted here for a laser plus static field as long as the ratio of field strengths is similar. [Note that if one uses as the second laser a harmonic of the fundamental laser, similar effects but of different physical origin (proportional to the sine of the phase difference between the two fields) are possible and will be discussed elsewhere.]

A static electric field or, alternatively, an additional lowintensity, linearly polarized laser beam permits significant control over the polarization properties of harmonics. Neither of the two predicted polarization effects occurs in the absence of the second field. Elliptic dichroism provides a unique case in the analysis of harmonic genera- tion of measuring an effect which depends on the sign of the helicity of an elliptically polarized laser beam. Either effect allows the direct determination of the interference between real and imaginary (dissipative) parts of the nonlinear susceptibilities, which is useful for distinguishing between different models of ionization and harmonic generation by atoms in strong fields.

This work was supported by NSF Grant No. PHY9722110, RFBR Grant No. 00-02-17843 and INTAS Grant No. 97-0369 (A. V.F., M. V.F., and N.L.M.), and the Alexander von Humboldt Foundation (D. B. M.).

*On leave from Institute for Space Sciences, Bucharest, Romania.

${ }^{\dagger}$ On leave from Faculty of Science and Mathematics, University of Sarajevo, Sarajevo, Bosnia-Herzegovina.

Present address: Max-Born Institute, Max-Born-Strasse 2a, 12489 Berlin, Germany.

[1] L.F. DiMauro and P. Agostini, Adv. At. Mol. Opt. Phys. 35, 79 (1995); P. Salières et al., ibid. 41, 83 (1999); M. Protopapas et al., Rep. Prog. Phys. 60, 389 (1997).

[2] F. A. Weihe et al., Phys. Rev. A 51, R3433 (1995).

[3] N. L. Manakov, Zh. Eksp. Teor. Fiz. 110, 1244 (1996) [Sov. Phys. JETP 83, 685 (1996)].

[4] N.H. Burnett et al., Phys. Rev. A 51, R3418 (1995).

[5] Ph. Antoine et al., Phys. Rev. A 55, 1314 (1997).

[6] M. Kakehata et al., Phys. Rev. A 55, R861 (1997); D. Schulze et al., ibid. 57, 3003 (1998).

[7] W. Becker et al., Phys. Rev. A 56, 645 (1997).

[8] J. Gao et al., J. Phys. B 32, 4153 (1999).

[9] M.Q. Bao and A.F. Starace, Phys. Rev. A 53, R3723 (1996).

[10] A. Lohr et al., Laser Phys. 7, 615 (1997).

[11] B. Wang et al., J. Phys. B 31, 1961 (1998).

[12] B. Wang et al., Phys. Rev. A 59, 2894 (1999).

[13] K. C. Kulander et al., in Super Intense Laser-Atom Physics, edited by B. Piraux et al., NATO ASI, Ser. B, Vol. 316 (Plenum, New York, 1993), p. 95; P. B. Corkum, Phys. Rev. Lett. 71, 1994 (1993).

[14] See, e.g., V. B. Berestetskii et al., Quantum Electrodynamics (Pergamon, Oxford, 1982), 2nd ed., Sec. 48; K. Blum, Density Matrix Theory and Applications (Plenum, New York, 1981), Sec. 1.2.5.

[15] S. I. Chu, Adv. At. Mol. Opt. Phys. 21, 197 (1985); N.L. Manakov et al., Phys. Rep. 141, 319 (1986).

[16] R. M. Potvliege and R. Shakeshaft, in Atoms in Intense Laser Fields, edited by M. Gavrila (Academic, New York, 1992), p. 373; Phys. Rev. A 40, 3061 (1989).

[17] N. Hokkyo, Prog. Theor. Phys. 33, 1116 (1965).

[18] N. L. Manakov and A. G. Fainshtein, Zh. Eksp. Teor. Fiz. 79, 751 (1980) [Sov. Phys. JETP 52, 382 (1980)].

[19] W. Becker et al., Phys. Rev. A 50, 1540 (1994).

[20] M. Yu. Kuchiev and V. N. Ostrovsky, Phys. Rev. A 59, 2844 (1999).

[21] Note that the effects predicted are invariant to spatial inversion of $\mathcal{F}$ (or $\mathbf{F}_{\Omega}$ ). As $\hat{\boldsymbol{e}}_{0} \rightarrow-\hat{\boldsymbol{e}}_{0}, \varphi \rightarrow \varphi+\pi$. This is equivalent in its effect on $\varphi$ to $\hat{\boldsymbol{\varepsilon}} \rightarrow-\hat{\boldsymbol{\varepsilon}}$. From Eqs. (1) and (2), $\hat{\boldsymbol{\varepsilon}} \rightarrow-\hat{\boldsymbol{\varepsilon}}$ is in turn equivalent to a phase change, $\omega t \rightarrow \omega t+\pi$, which does not affect the predictions (provided the two laser frequencies are incommensurate). 